\title{
Analysis of Flexibility of Proteins by means of Positive and Negative Ion MALDI In-Source Decay Mass Spectrometry
}

\author{
Ryunosuke Iimuro and Mitsuo Takayama* \\ Graduate School of Nanobioscience, Mass Spectrometry Laboratory, Yokohama City University
}

\begin{abstract}
The amino acid residues susceptible to in-source decay (ISD) in matrix-assisted laser desorption/ionization (MALDI) mass spectrometry have been identified from both positive and negative ion ISD spectra of cytochrome $c$, myoglobin, thioredoxin and bovine serum albumin. Backbone cleavages at the $\mathrm{N}-\mathrm{C}_{\alpha}$ bonds of $\mathrm{Xxx}-\mathrm{Asp}, \mathrm{Xxx}-\mathrm{Asn}, \mathrm{Xxx}-\mathrm{Cys}$, and Gly-Xxx residues gave discontinuous intense peaks of c-ions, independent of positive and negative ion mode. The intensity values for c-ions, Int(c), were defined to allow estimation of the discontinuous intense peaks of c-ions. The identities of the high intensity value residues Asp, Asn, Cys, and Gly were compared with those identified using other measures of flexibility such as the B-factor, turn preferential factor and protection factor. The comparison indicates that Asp, Asn, and Gly residues are common to all measures. Thus, the intensity values of c-ions can be adopted as a measure of protein flexibility.
\end{abstract}

Please cite this article as: R. Iimuro and M. Takayama, Analysis of Flexibility of Proteins by means of Positive and Negative Ion MALDI In-Source Decay Mass Spectrometry, Mass Spectrom (Tokyo) 2014; 3(2): S0023; DOI: 10.5702/massspectrometry.S0023

Keywords: matrix-assisted laser desorption/ionization, in-source decay, protein, flexibility

(Received September 4, 2013; Accepted November 19, 2013)

\section{INTRODUCTION}

Matrix-assisted laser desorption/ionization mass spectrometry (MALDI MS) ${ }^{1,2)}$ is an indispensable powerful method for studying thermally labile bio-polymers such as proteins, nucleic acids and saccharides. MALDI MS presents a unique and specific cleavage method namely "in-source decay (ISD)"3) as a top-down tool for proteomics. ${ }^{4,5)}$ Recent applications of MALDI-ISD include its use for identifying protein isoforms ${ }^{6}$ and disease biomarkers ${ }^{4)}$ and for imaging analysis. ${ }^{7)}$ The most useful aspect of MALDI-ISD is that it produces a lot of fragment ions that reflect sequence information of intact proteins without any need for digestion.

Another aspect of MALDI-ISD that has attracted interest is its application to studying the backbone flexibility of proteins by means of hydrogen/deuterium exchange (HDX) with nuclear magnetic resonance (NMR) spectroscopy. ${ }^{8)}$ In a previous work, we reported that amino acid residues susceptible to ISD (Asp, Asn, and Gly) in equine cytochrome $c$ were partly consistent with them being in flexible regions as identified by NMR-HDX. ${ }^{9)}$ MALDI-ISD can measure the flexibility of proteins by means of the interaction between carbonyl oxygens on the peptide backbone and hydrogen radicals from matrix molecules, while the flexibility in NMR-HDX can be measured by the rate of HDX reaction between backbone amide hydrogens and environmental water molecules. The flexible amino acid residues identified from the NMR-HDX of ubiquitin and cytochrome $c$ have been reported to be Asp, Asn, Gly, Lys, Thr, Ile, and Met. ${ }^{10,11)}$ In MALDI-ISD the flexibility of amino acid residues can be measured by the discontinuous intense peaks of c-ions originating from cleavage at the $\mathrm{N}-\mathrm{C}_{\alpha}$ bond of the backbone. ${ }^{12,13)}$ The flexible Asp, Asn, and Gly residues identified by means of MALDI-ISD are also those that prefer a secondary turn structure. ${ }^{13)}$

It is also of interest that flexible proteins that are intrinsically disordered have been recognized to be key factors in the intermolecular interactions with other proteins, nucleic acids and drug molecules. ${ }^{14)}$ The flexibility of protein molecules can be measured using several parameters such as the B-factor based on the X-ray crystallography, ${ }^{15,16)}$ the turn preferential factor, ${ }^{17,18)}$ and the protection factor based on NMR-HDX. ${ }^{11)}$ Understanding the flexibility of proteins is of importance from the standpoints of basic and practical interests.

Here we identify amino acid residues susceptible to ISD in both positive and negative ion MALDI-ISD of equine cytochrome $c$, equine myoglobin, human thioredoxin and bovine serum albumin (BSA), on the basis of the peak intensity of c-ions which are amino $(\mathrm{N})$-terminal side fragments originating from the cleavage at the $\mathrm{N}-\mathrm{C}_{\alpha}$ bonds of the peptide backbone. The susceptibility for each amino acid residue in those proteins was estimated from the intensity values of c-ions, Int(c), observed in the ISD spectra. ${ }^{9)}$ We show that in both positive and negative ion MALDI-ISD of

* Correspondence to: Mitsuo Takayama, Yokohama City University, 22-2 Seto, Kanazawa-ku, Yokohama 236-0027, Japan, e-mail: takayama@yokohama-cu.ac.jp Abbreviations: MALDI (matrix-assisted laser desorption/ionization), ISD (in-source decay), 5,1-ANL (5-amino-1-naphthol), HDX (hydrogen/deuterium exchange) The contents of this paper were presented at 4th AOMSC Taiwan 2013 


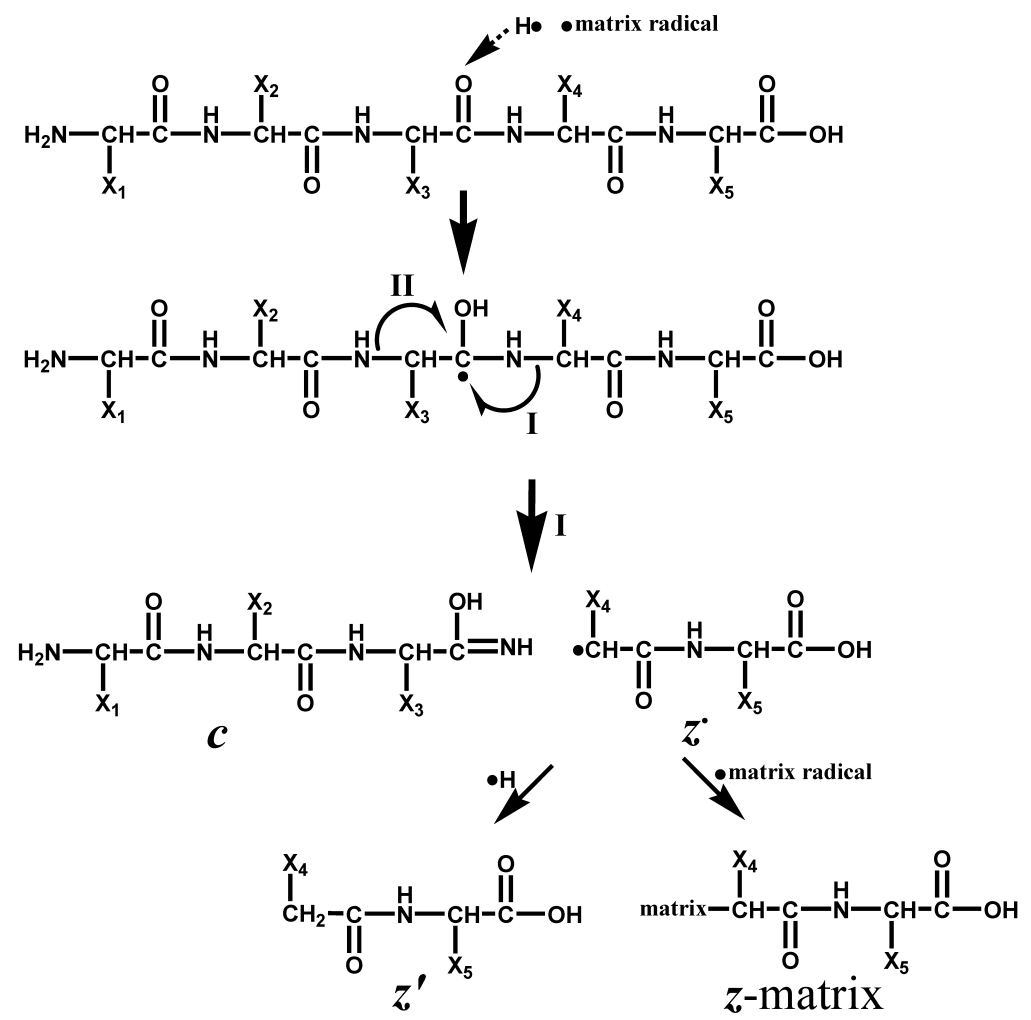

(a)

Scheme 1. The formation mechanism of MALDI-ISD fragments $c, z^{\prime}$, and $z$-matrix.

proteins Asp, Asn, Gly, and Cys residues are more susceptible than the rest. The susceptible Asp, Asn, Gly, and Cys residues are compared to the flexible residues identified by means of the B-factor, ${ }^{19)}$ the turn preferential factor ${ }^{20)}$ and the protection factors..$^{10,11)}$

\section{EXPERIMENTAL}

\section{Materials}

The matrix 5-amino-1-naphthol (5,1-ANL) was purchased from Tokyo Chemical Industry (Tokyo, Japan). Acetonitrile was purchased from Wako Pure Chemical Industries, Ltd. (Osaka, Japan). Water used in all experiments was purified using a MilliQ water purification system from Millipore (Billerica, MA, USA). Equine holo-cytochrome $c$ $\left(M_{\mathrm{r}}\right.$ 12360.4), equine apo-myoglobin $\left(M_{\mathrm{r}} 16951.4\right)$, human thioredoxin with His-tag $\left(M_{\mathrm{r}}\right.$ 13769.6) and bovine serum albumin $\left(M_{\mathrm{r}}\right.$ 66430.3) were purchased from Sigma-Aldrich (Saint Louis, MO, USA). All reagents were used without further purification.

\section{Protein and matrix preparations}

For the MALDI-ISD experiments, analyte protein was dissolved in water at a concentration of $20 \mathrm{pmol} / \mu \mathrm{L}$. The matrix material was dissolved in water-acetonitrile $(3: 7, \mathrm{v} / \mathrm{v})$ without any additives. A sample solution was prepared by mixing a volume of $10 \mu \mathrm{L}$ of analyte solution with a volume of $10 \mu \mathrm{L}$ of matrix solution. A volume of $1.0 \mu \mathrm{L}$ of the sample solution was deposited onto a stainless-steel MALDI plate and the solvents were removed by allowing evaporation in air at room temperature.

\section{MALDI MS}

MALDI-ISD spectra were acquired on a time-of-flight mass spectrometer AXIMA-CFR (Shimadzu, Kyoto, Japan) equipped with a nitrogen laser ( $337 \mathrm{~nm}$ wavelength) operating at a pulse rate of $10 \mathrm{~Hz}$. The pulse width of the laser was $4 \mathrm{~ns}$. The laser spot size on the target substrate was $c a$. $100 \mu \mathrm{m}$ in diameter. The ions generated by MALDI were accelerated using $20 \mathrm{kV}$ with delayed extraction. The analyzer was operated in linear mode and the ions were detected using a microchannel plate detector. A total of 500 shots were accumulated for each mass spectrum acquisition. The reproducibility of each ISD spectrum was confirmed by the fragment intensity patterns for several measurements using the raster function installed on the AXIMA-CFR mass spectrometer.

\section{RESULTS AND DISCUSSION}

\section{Positive and negative ion MALDI-ISD spectra of intact proteins}

The formation of the ISD fragments is shown in Scheme $1 .^{21,22)}$ In the processes (b) and (c) in Scheme 1, it can be confirmed that cleavage I is energetically more favorable than reaction II, by using a molecular orbital (MO) calculation (Gaussian 09 with B3LYP/6-31+G(d)). The MO calculation for a model peptide Ala-Ala-Ala suggested that the Gibbs energies $\Delta G(\mathrm{~kJ} / \mathrm{mol})$ for reactions I and II were -90.84 and +59.17 , respectively (detailed calculation procedures not shown). In Scheme 1, the even electron closed shell fragments $z^{\prime}$ and $z$-matrix can be formed by collisions of the odd electron radical species $z^{\cdot}$ with hydrogen or matrix radicals in a dense-gas MALDI plume within several tens of $\mathrm{ns}$ in the ion source. The ionization (protonation and deprotonation) of the fragments $c, z^{\prime}$ and $z$-matrix also takes place in the plume simultaneously and independently of the ISD processes. ${ }^{23)}$ From the nomenclature for the 
ISD spectra of equine cytochrome $c$
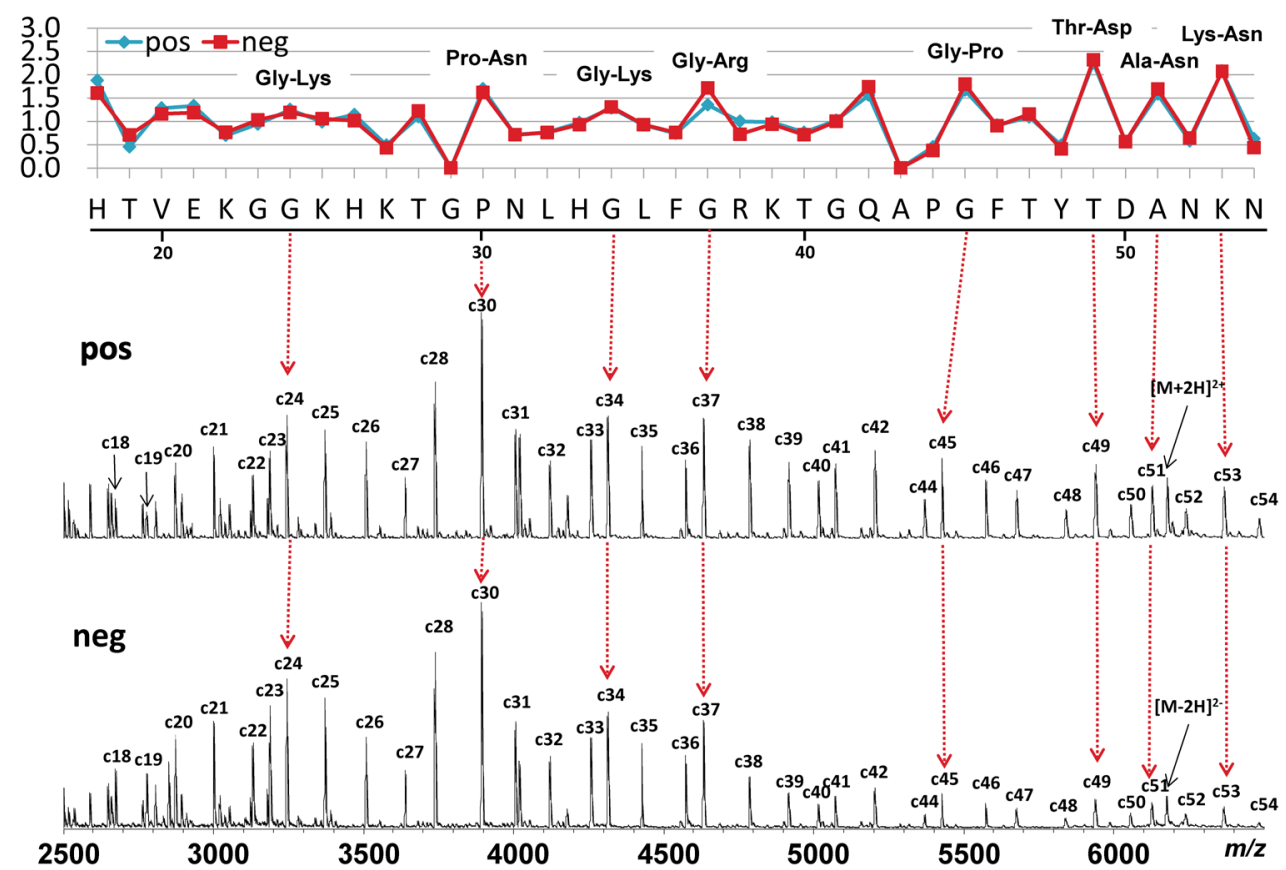

Fig. 1. Positive and negative ion MALDI-ISD spectra of equine holo-cytochrome $c$ obtained with 5,1-ANL matrix. Intensity values (upper) estimated for $\mathrm{c}_{18}$ to $\mathrm{c}_{54}$ ions.

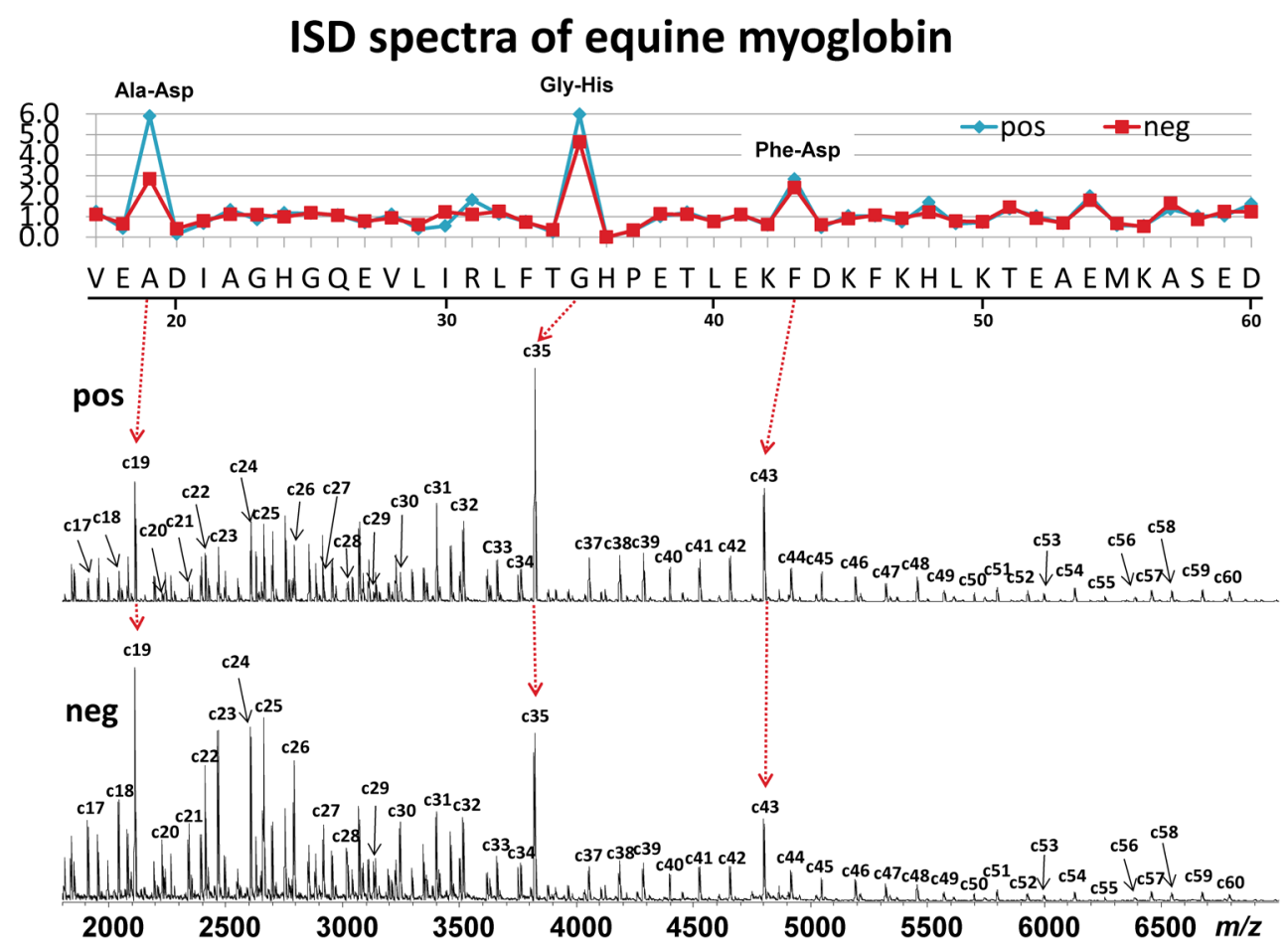

Fig. 2. Positive and negative ion MALDI-ISD spectra of equine apo-myoglobin obtained with 5,1-ANL matrix. Intensity values (upper) estimated for $\mathrm{c}_{17}$ to $\mathrm{c}_{60}$ ions.

ISD fragments in Scheme 1, singly charged positive and negative ions can be observed as follows, i.e., $\mathrm{c}$-ion $=[\mathrm{c}+\mathrm{H}]^{+}$, $z^{\prime}$-ion $=\left[z^{\prime}+\mathrm{H}\right]^{+}$, and $z$-matrix ion $=[z \text {-matrix }+\mathrm{H}]^{+}$for positive mode, and $\mathrm{c}$-ion $=[\mathrm{c}-\mathrm{H}]^{-}, z^{\prime}$-ion $=\left[z^{\prime}-\mathrm{H}\right]^{-}$, and $z$-matrix ion $=[z \text {-matrix }-\mathrm{H}]^{-}$for negative mode.

Positive and negative ion MALDI-ISD spectra of cytochrome $c$, myoglobin, thioredoxin and BSA were obtained with 5,1-ANL matrix, ${ }^{9,13,24)}$ as shown in Figs. 1-4. The ISD spectra shown here are partial spectra showing mainly cions to allow estimation of the intensity values for $\mathrm{c}$-ions as follows. ${ }^{9)}$

$$
\operatorname{Int}\left(\mathrm{c}_{n}\right)=\operatorname{Int}\left(\mathrm{c}_{n}\right) /\left(\operatorname{Int}\left(\mathrm{c}_{n-1}\right)+\operatorname{Int}\left(\mathrm{c}_{n+1}\right)\right) / 2
$$

The intensity value for an $n$-th $c$-ion, $\operatorname{Int}\left(\mathrm{c}_{n}\right)$, can be defined by the ratio of the intensity of the $c_{n}$-ion peak to the average intensity of the adjacent side peaks. Both posi- 


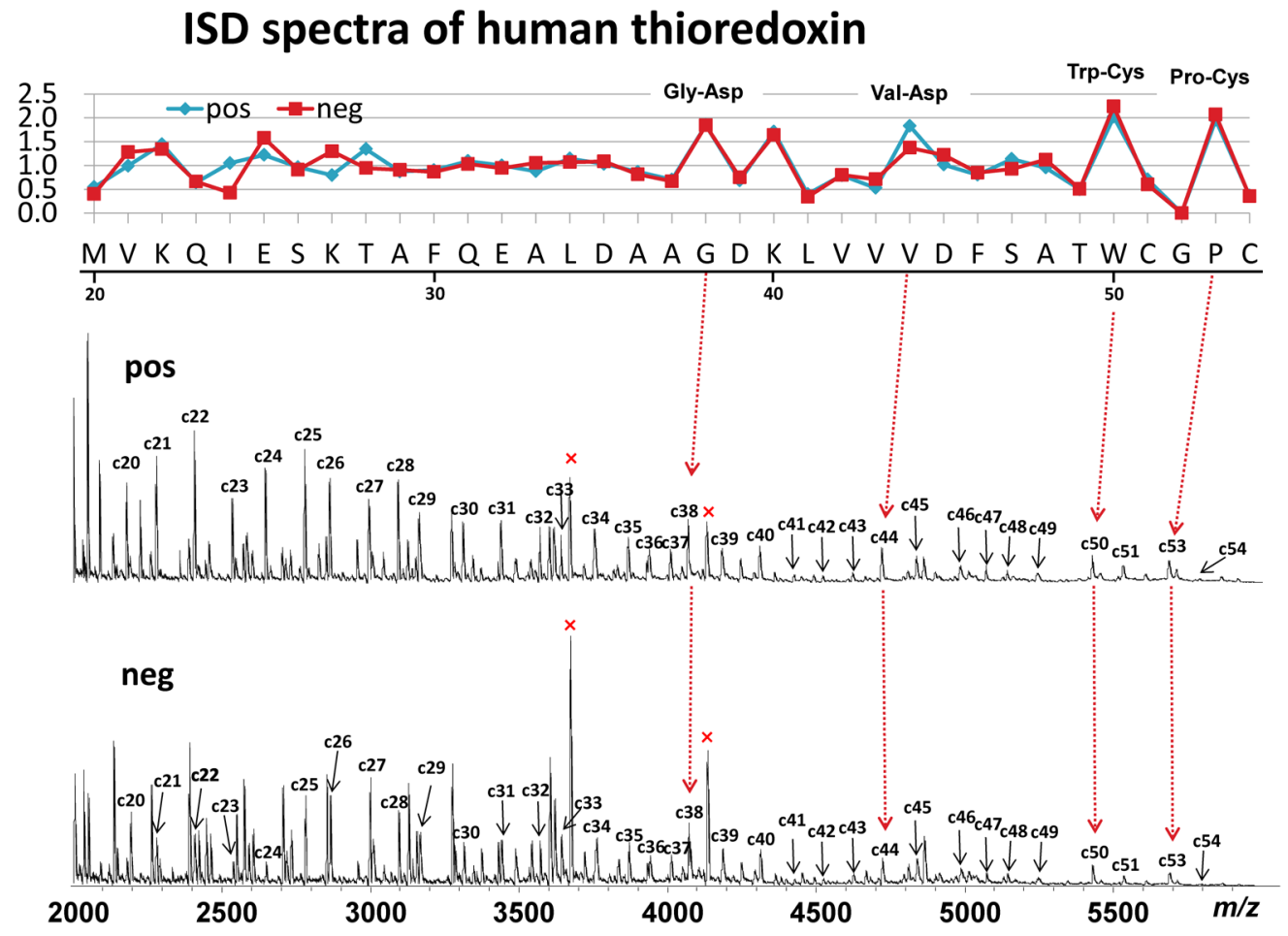

Fig. 3. Positive and negative ion MALDI-ISD spectra of human thioredoxin obtained with 5,1-ANL matrix. Intensity values (upper) estimated for $c_{20}$ to $c_{54}$ ions. The peaks indicated by asterisk could not be assigned.

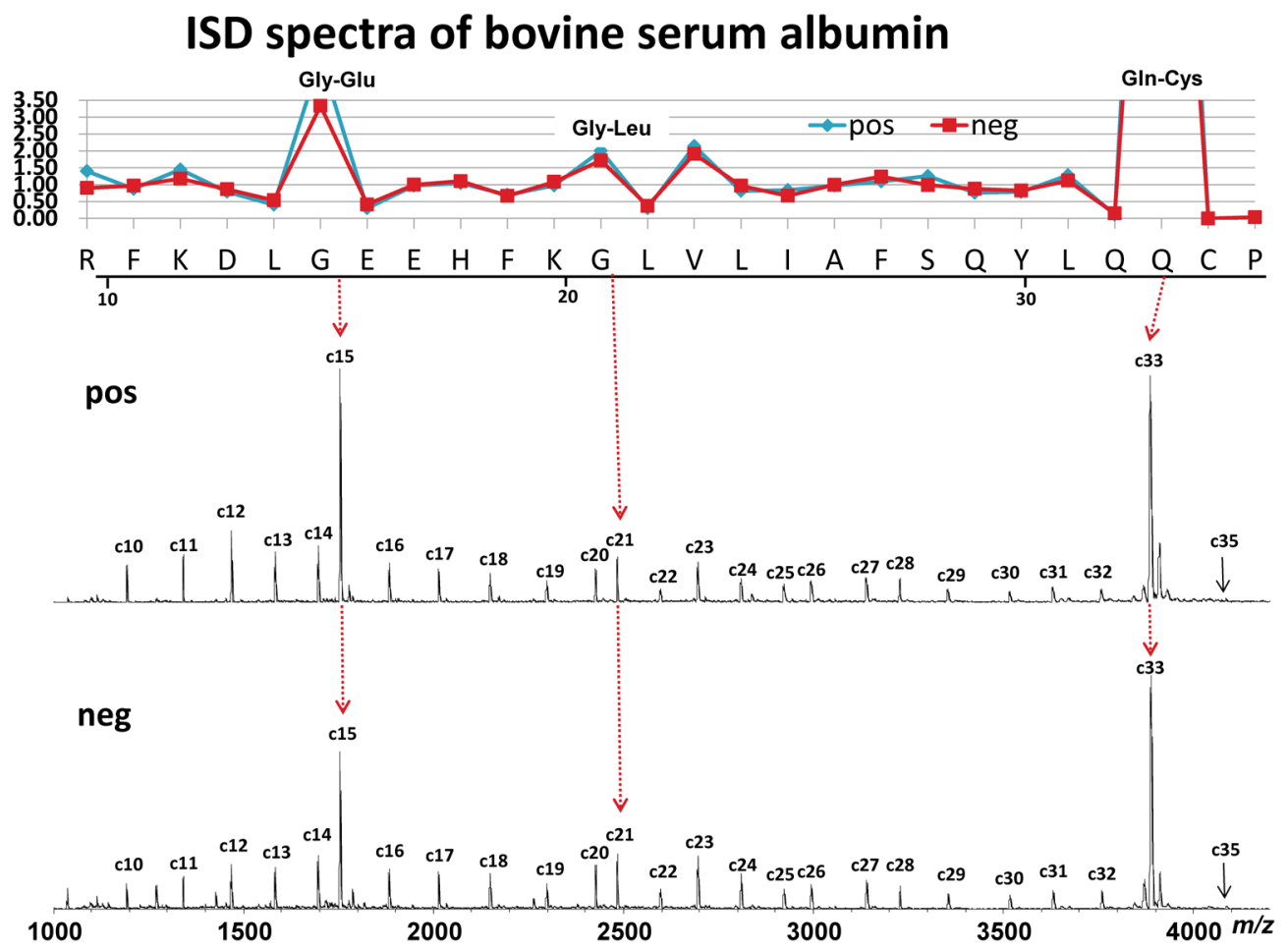

Fig. 4. Positive and negative ion MALDI-ISD spectra of bovine serum albumin obtained with 5,1-ANL matrix. Intensity values (upper) estimated for $\mathrm{c}_{10}$ to $\mathrm{c}_{35}$ ions.

tive and negative ion MALDI-ISD spectra for each protein showed $z^{\prime}$-ions, and $c$-ions as well. The $z^{\prime}$-ions are carboxyl (C)-terminal side fragment ions appearing in relatively low $\mathrm{m} / \mathrm{z}$ regions (data not shown).

In Figs. 1-4, the intensity values estimated for both positive and negative c-ions are shown by blue and red lines in the upper portion of the figure. The ISD fragment patterns were very similar to each other in terms of positive and negative c-ion abundance for all the analyte proteins. In other words, the ISD spectra show common properties with respect to discontinuous intense c-ion peaks in both ion modes. 
In the MALDI-ISD spectra of cytochrome $c$ (Fig. 1), discontinuous intense fragment peaks (cleavage site) were observed at $c_{24}$ (Gly-Lys), $c_{30}$ (Pro-Asn), $c_{34}$ (Gly-Lys), $c_{37}$

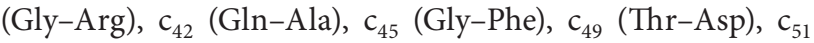
(Ala-Asn), and $c_{53}$ (Lys-Asn) in both positive and negative ions. The C-terminal side $z_{35}^{\prime}$ (Glu69-Asn70), and $z_{35}$-matrix ions were also observed in both ion modes, as was previously reported in positive mode, ${ }^{9)}$ although here we did not assign the peaks of $z^{\prime}$-ions and $z$-matrix ions. In the ISD spectra of myoglobin (Fig. 2), the common striking discontinuous intense peaks were observed at $c_{19}$ (Ala-Asp), $c_{35}$ (Gly-His), and $\mathrm{c}_{43}$ (Phe-Asp) in both ion modes. In the ISD spectra of thioredoxin (Fig. 3), the common discontinuous intense peaks were observed at $c_{38}$ (Gly-Asp), $c_{40}$ (Lys-Leu), $\mathrm{c}_{44}$ (Val-Asp), $\mathrm{c}_{50}$ (Trp-Cys), and $\mathrm{c}_{53}$ (Pro-Cys) in both ion modes. The ISD spectra of BSA (Fig. 4) showed two striking discontinuous intense peaks of $c_{15}(\mathrm{Gly}-\mathrm{Glu})$, and $\mathrm{c}_{33}$ (GlnCys) in both modes.

The intensity values estimated from positive and negative ion ISD data for each protein were compatible to each other, except for the $c_{24}$ to $c_{28}$ ions of thioredoxin (Fig. 3). This indicates that the susceptibility of amino acid residues in proteins to ISD is independent of MALDI ionization (protonation and deprotonation), as shown in Scheme 1. It should be noted, however, that in general the peak abundance of ISD fragment ions may be dependent upon two factors: 1) the susceptibility of amino acid residues to ISD and 2) the sites of charge due to the presence or absence of acidic or basic residues. We know that there is a general tendency for fragments including basic sites (Arg, Lys, His) or the amino $(\mathrm{N})$-terminus to give relatively abundant positive ion peaks, while those containing acidic sites (Glu, Asp or the C-terminus) give relatively intense negative ion peaks. The results described above concerning the intensity values imply that protein molecules are without large deviations toward basic or acidic charge states, so that the peak abundance of ISD fragment ions entirely depends on the susceptibility to ISD.

\section{Average intensity values estimated from ISD data of four different proteins}

The intensity values for each amino acid residue averaged with all combinations of $\mathrm{N}$-terminal side pair $\mathrm{Xxx}_{\mathrm{x}}-\mathrm{X}_{n}$ or C-terminal side pair $\mathrm{X}_{n}-\mathrm{Xxx}$ for $n$-th residue $\mathrm{X}_{n}$ in the four different proteins described above are summarized in Table 1. A maximum intensity value of 2.0 (asterisk in Table 1) was set for the residues having intensity values over 2.0 such as $\mathrm{c}_{49}$ (Thr-Asp) in cytochrome $c$ (Fig. 1), $\mathrm{c}_{19}$ (Ala-Asp), $\mathrm{c}_{35}$ (Gly-His), and $\mathrm{c}_{43}$ (Phe-Asp) in myoglobin (Fig. 2), $\mathrm{c}_{50}$ (Trp-Cys) in thioredoxin (Fig. 3), and $c_{15}$ (Gly-Glu), and $c_{33}$ (Gln-Cys) in BSA (Fig. 4). The intensity values over 2.0 for Trp-Xxx (pos and neg) and Xxx-Met (neg) in Table 1 were estimated from only one Trp-Cys pair for thioredoxin and Glu-Met for myoglobin, so that the values 2.0 for Trp-Xxx and $\mathrm{Xxx}-\mathrm{Met}$ are lacking in reliability. The intensity value of zero for $\mathrm{Xxx}$-Pro is due to incomplete cleavage of the backbone. For the estimation of the intensity values in Eq. (1), Int $\left(c_{n-2}\right)$ or Int $\left(c_{n+2}\right)$ was used when Int $\left(c_{n-1}\right)$ or Int $\left(c_{n+1}\right)$ was corresponding to $\mathrm{Xxx}$-Pro residue. Further, the intensity values of $\mathrm{c}_{42}(\mathrm{Gln}-\mathrm{Ala})$ for myoglobin (Fig. 1 and Table 2) and $c_{40}$ (Lys-Leu) for thioredoxin (Fig. 3 and Table 2) were over-estimation, because $\mathrm{Xxx}-\mathrm{Gly} 45$ (Fig. 1) and $\mathrm{Xxx}-\mathrm{Val} 42$
Table 1. Average intensity values of c-ions, Int(c), estimated from positive and negative ion MALDI-ISD spectra of cytochrome $c$, myoglobin, thioredoxin and BSA with 5,1-ANL matrix.

\begin{tabular}{ccccc}
\hline $\begin{array}{c}\text { Pos } \\
\mathrm{Xxx}_{n}\end{array}$ & $\begin{array}{c}\mathrm{Neg} \\
\mathrm{Xxx}-\mathrm{X}_{n}\end{array}$ & $\mathrm{X}_{n}$ & $\begin{array}{c}\text { Pos } \\
\mathrm{X}_{n}-\mathrm{Xxx}\end{array}$ & $\begin{array}{c}\text { Neg } \\
\mathrm{X}_{n}-\mathrm{Xxx}\end{array}$ \\
\hline $2.00^{*}$ & $2.00^{*}$ & Asn & 0.64 & 0.62 \\
1.38 & 1.36 & Asp & 0.80 & 0.85 \\
0.84 & 0.81 & Gly & 1.28 & 1.32 \\
$2.00^{*}$ & $2.00^{*}$ & Cys & 0.60 & 0.55 \\
0.58 & 0.62 & Val & 1.18 & 1.11 \\
0.63 & 0.68 & Ile & 0.76 & 0.80 \\
0.96 & 1.33 & Arg & 1.26 & 0.91 \\
0.92 & 0.86 & Ala & 1.06 & 1.08 \\
1.08 & 0.97 & Glu & 1.00 & 1.04 \\
1.02 & 1.00 & Gln & 1.02 & 1.02 \\
0.93 & 0.90 & Lys & 0.97 & 0.99 \\
1.03 & 1.03 & Phe & 0.98 & 0.95 \\
0.88 & 0.94 & Thr & 0.98 & 0.98 \\
1.18 & 1.13 & Leu & 0.81 & 0.82 \\
0.97 & 1.05 & His & 1.17 & 1.12 \\
1.10 & 1.27 & Ser & 1.03 & 0.92 \\
0.00 & 0.00 & Pro & 1.00 & 1.00 \\
1.29 & $2.00^{*}$ & Met & 0.59 & 0.58 \\
1.03 & 1.02 & Tyr & 0.68 & 0.66 \\
0.5 & 0.5 & Trp & $2.00^{*}$ & $2.00^{*}$ \\
\hline
\end{tabular}

*Intensity values over 2.0.

Table 2. Discontinuous intense c-ions and cleavage sites observed in positive and negative ion ISD spectra of cytochrome $c$, myoglobin, thioredoxin, and BSA.

\begin{tabular}{lc}
\hline \multicolumn{1}{c}{ Protein } & Discontinuous intense c-ions and cleavage sites \\
\hline Cytochrome $c$ & $\mathrm{c}_{24}$ (Gly-Lys), $\mathrm{c}_{30}$ (Pro-Asn), $\mathrm{c}_{34}$ (Gly-Lys), $\mathrm{c}_{37}$ (Gly- \\
& Arg), $\mathrm{c}_{42}(\mathrm{Gln}-\mathrm{Ala}), \mathrm{c}_{45}$ (Gly-Phe), $\mathrm{c}_{49}$ (Thr-Asp), \\
& $\mathrm{c}_{51}$ (Ala-Asn), $\mathrm{c}_{53}$ (Lys-Asn) \\
Myoglobin & $\mathrm{c}_{19}$ (Ala-Asp), $\mathrm{c}_{35}$ (Gly-His), $\mathrm{c}_{43}$ (Phe-Asp) \\
Thioredoxin & $\mathrm{c}_{38}$ (Gly-Asp), $\mathrm{c}_{40}$ (Lys-Leu), $\mathrm{c}_{44}$ (Val-Asp), \\
& $\mathrm{c}_{50}$ (Trp-Cys), $\mathrm{c}_{53}$ (Pro-Cys) \\
BSA & $\mathrm{c}_{15}$ (Gly-Glu), $\mathrm{c}_{33}$ (Gln-Cys) \\
\hline
\end{tabular}

Table 3. Flexible amino acid residues of protein estimated from four different measures of flexibility.

\begin{tabular}{ll}
\hline \multicolumn{1}{c}{ Factor for flexibility } & \multicolumn{1}{c}{ Flexible amino acid residue } \\
\hline Intensity value (this study) & Asp, Asn, Gly, Cys \\
B-Factor $^{19)}$ & Asp, Asn, Gly, Pro, Lys, Glu, Gln, Ser \\
Turn preference $^{20)}$ & Asp, Asn, Gly, Pro, Ser \\
Protection factor $^{10,11)}$ & Asp, Asn, Gly, Lys, Thr, Ile, Met \\
\hline
\end{tabular}

(Fig. 3) were less susceptible for ISD.9) This is supported from the data of average intensity values for $\mathrm{Gln}-\mathrm{Xxx}$, $\mathrm{Xxx}-\mathrm{Ala}$, Lys-Xxx, and Xxx-Leu in Table 1. So that, Table 1 indicates that $\mathrm{Xxx}-\mathrm{Asn}, \mathrm{Xxx}-\mathrm{Asp}, \mathrm{Xxx}-\mathrm{Cys}$, and Gly-Xxx residues are more susceptible than the rest independent of positive and negative ion mode.

\section{Comparison of several different factors for mea- suring flexibility of amino acid residues in protein}

The intensity values of the discontinuous intense c-ions and cleavage sites shown in the upper part of Figs. 1-4, are summarized in Table 2. Of the eighteen c-ions shown in Table 2, the common cleavage sites of Gly-Xxx, Xxx-Asp, $\mathrm{Xxx}-\mathrm{Asn}$, and $\mathrm{Xxx}-\mathrm{Cys}$ account for seven, five, three, and three c-ions, respectively. Gly, the highest frequency residue, is known as the most flexible residue preferred in turn 


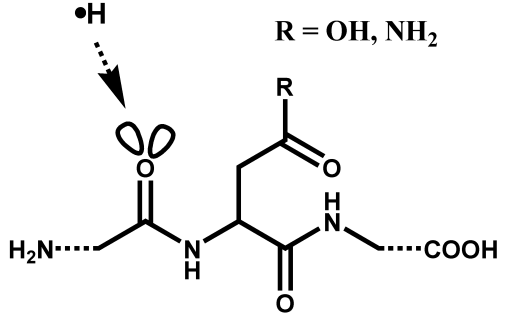

Xxx-Asp/Asn

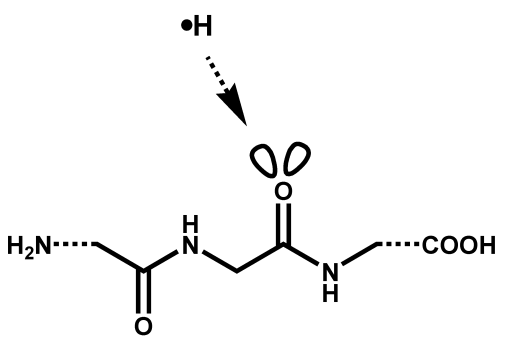

Gly-Xxx

Scheme 2. Preferential access of hydrogen radicals to carbonyl oxygens of the backbone.

structures. $^{20)}$ Asp and Asn residues are also preferred in flexible turn structures, while the Cys residue is preferred in $\beta$-sheet. ${ }^{20)}$ The turn structure can be classified as a flexible secondary structure.

Here we compare the flexibility of the amino acid residues by means of the intensity value Int(c) described above with several other factors representing the flexibility of proteins, such as the B-factor, ${ }^{19)}$ the turn preferential factor ${ }^{20)}$ and the protection factor, ${ }^{10,11)}$ as summarized in Table 3 . It is therefore of interest that Asp, Asn, and Gly residues are identified as flexible by all the measures of flexibility despite the different principles used in the different measures. From reports of the secondary structure preferences of amino acid residues, ${ }^{20)}$ Asp, Asn, and Gly residues are preferred in flexible turn structures rather than in intra-molecular hydrogen-bonded structures such as $\alpha$-helix and $\beta$-sheet. In particular, the Gly residue tends to destroy the formation of helix structure. As previously reported, ${ }^{12,13)}$ the helix structure may protect backbone carbonyl oxygens from binding of hydrogen radicals (as in Scheme 1). This suggests that the carbonyl oxygens on the N-terminal side of Asp and Asn residues (Xxx-Asp/Asn) and those on the C-terminal side of Gly residues (Gly-Xxx) would be expected to be more exposed to hydrogen radicals or matrix molecules than other residues (Scheme 2).

Although it is difficult to estimate the conformations and secondary structures of protein molecules in/on MALDI matrix crystals, the high susceptibility of Asp, Asn, Gly, and Cys residues to both positive and negative ion ISD suggests that the intensity values in MALDI-ISD could be used as a factor for describing protein flexibility. The intensity values estimated from the MALDI-ISD fragment ions is based on the interactions between backbone carbonyl oxygens and hydrogen or matrix molecules in the MALDI crystal phase or dense-gas plume. ${ }^{9)}$ The high susceptibility of amino acid residues to ISD means that there is preferential access of hydrogen radicals to the carbonyl oxygens of the peptide backbone, as shown in Scheme 2. This indicates that the carbonyl oxygens in the susceptible amino acid residues are exposed to hydrogen radicals or environmental matrix molecules as a hydrogen donor.

\section{CONCLUSION}

Amino acid residues susceptible to MALDI-ISD, which gives discontinuous intense peaks of c-ions originating from cleavage at the $\mathrm{N}-\mathrm{C}_{\alpha}$ bonds of the peptide backbone, have been identified in both positive and negative ion MALDIISD spectra of cytochrome $c$, myoglobin, thioredoxin, and
BSA. The results obtained here show that the $\mathrm{N}-\mathrm{C}_{\alpha}$ bonds at $\mathrm{Xxx}-\mathrm{Asp}, \mathrm{Xxx}-\mathrm{Asn}, \mathrm{Xxx}-\mathrm{Cys}$, and Gly-Xxx residues gave relatively high intensity values for $c$-ions with $\operatorname{Int}(\mathrm{c})$ used as a measure of the discontinuous intense peaks in the ISD spectra independent of positive and negative ion mode. The residues Asp, Asn, Cys, and Gly susceptible to ISD were compared with residues estimated from other measures of protein flexibility, such as the B-factor, the turn preferential factor, and the protection factor. The comparison showed that Asp, Asn, and Gly residues were common to all the measures, in spite of the different principles used to measure them. This implies that the intensity values of c-ions could be adopted as a factor for measuring protein flexibility.

\section{Acknowledgements}

The paper was supported by the fund for Creation of Innovation Centers for Advanced Interdisciplinary Research Area Program in the Project for Developing Innovation Systems from the Ministry of Education, Culture, Sports, Science and Technology of Japan.

\section{REFERENCES}

1) M. Karas, D. Bachmann, U. Bahr, F. Hillenkamp. Matrix-assisted ultraviolet laser desorption of non-volatile compounds. Int. J. Mass Spectrom. Ion Process. 78: 53-68, 1987.

2) K. Tanaka, H. Waki, Y. Ido, S. Akita, Y. Yoshida, T. Yoshida, T. Matsuo. Protein and polymer analyses up to $\mathrm{m} / \mathrm{z} 100000$ by laser ionization time-of-flight mass spectrometry. Rapid Commun. Mass Spectrom. 2: 151-153, 1988.

3) R. S. Brown, J. J. Lennon. Sequence-specific fragmentation of matrix-assisted laser-desorped protein/peptide ions. Anal. Chem. 67: 3990-3999, 1995.

4) A. Resemann, D. Wunderlich, U. Rothbauer, B. Warscheid, H. Leonhardt, J. Fuchser, K. Kuhlmann, D. Suckau. Top-down de novo protein sequencing of a $13.6 \mathrm{kDa}$ Camelid single heavy chain antibody by matrix-assisted laser desorption/ionizationtime-of-flight/time-of-flight mass spectrometry. Anal. Chem. 82: 3283-3292, 2010.

5) D. Calligaris, C. Villard, D. Lafitte. Advances in top-down proteomics for disease biomarker discovery. J. Proteomics 10: 920-934, 2011.

6) R. Ait-Belkacem, D. Calligaris, L. Sellami, C. Villard, S. Granjeaud, T. Schembri, C. Berenguer, L. Ouafik, D. Figarella-Branger, O. Chinot, D. Lafitte. Tubulin isoforms identified in the brain by MALDI in-source decay. J. Proteome 79: 172-179, 2013.

7) D. Debois, V. Bertrand, L. Quinton, M.-C. De Pauw-Gillet, E. De Pauw. MALDI-in source decay applied to mass spectrometry imaging: A new tool for protein identification. Anal. Chem. 82: 4036-4045, 2010. 
8) K. D. Rand, N. Bache, M. M. Nedertoft, J. D. Jorgensen. Spatially resolved protein hydrogen exchange measured by matrix-assisted laser desorption ionization in-source decay. Anal. Chem. 83: 8859-8862, 2011.

9) M. Takayama. Flexible $\mathrm{Xxx}-\mathrm{Asp} / \mathrm{Asn}$ and Gly-Xxx residues of equine cytochrome $c$ in matrix-assisted laser desorption/ionization in-source decay mass spectrometry. Mass Spectrom (Tokyo) 1: A0007, 2012

10) J. S. Milne, L. Mayne, H. Roder, A. J. Wand, S. W. Englander. Determinations of protein hydrogen exchange studied in equine cytochrome c. Protein Sci. 7: 739-745, 1998.

11) C. Bougault, L. Feng, J. Glushka, E. Kupce, J. H. Prestegard. Quantitation of rapid proton-deuteron amide exchange using hadamard spectroscopy. J. Biomol. NMR 28: 385-390, 2004.

12) M. Takayama. Susceptible region of myoglobins to in-source decay using matrix-assisted laser desorption/ionization coupled with delayed extraction reflectron time-of-flight mass spectrometer. J. Mass Spectrom. Soc. Jpn. 50: 304-310, 2002.

13) M. Takayama, I. Osaka, M. Sakakura. Influence of secondary structure on in-source decay of protein in matrix-assisted laser desorption/ionization mass spectrometry. Mass Spectrom (Tokyo) 1: A0001, 2012.

14) V. N. Uversky, A. K. Dunker. Understanding protein non-folding. Biochim. Biophys. Acta 1804: 1231-1264, 2010.

15) P. A. Karplus, G. E. Schulz. Prediction of chain flexibility in proteins. Naturwiss. 72: 212-213, 1985.
16) P. Radivojac, Z. Obradoic, D. K. Smith, G. Zhu, S. Vucetic, C. J. Brown, J. D. Lawson, A. K. Dunker. Protein flexibility and intrinsic disorder. Protein Sci. 13: 71-80, 2004.

17) P. Y. Chow, G. D. Fasman. Prediction of b-turns. Biophys. J. 26: 367-384, 1979.

18) F. Huang, W. M. Nau. A conformational flexibility scale for amino acids in proteins. Angew. Chem. Int. Ed. 42: 2269-2272, 2003.

19) D. K. Smith, P. Radivojac, Z. Obradovic, A. K. Dunker, G. Zhu. Improved amino acid flexibility parameters. Protein Sci. 12: 1060-1072, 2003.

20) R. W. Williams, A. Chang, D. Juretic, S. Loughran. Secondary structure predictions and medium range interactions. Biochim. Biophys. Acta 916: 200-204, 1987.

21) M. Takayama. $\mathrm{N}-\mathrm{C}_{\alpha}$ bond cleavage of the peptide backbone via hydrogen abstraction. J. Am. Soc. Mass Spectrom. 12: 1044-1049, 2001 .

22) K. Demeure, V. Gabelica, E. A. De Pauw. New advances in the understanding of the in-source decay in MALDI-TOF-MS. J. Am. Soc. Mass Spectrom. 21: 1906-1917, 2010.

23) M. Takayama, A. Tsugita. Does in-source decay occur independent of the ionization process in matrix-assisted laser desorption? Int. J. Mass Spectrom. 181: L1-L6, 1998.

24) I. Osaka, M. Sakai, M. Takayama. 5-Amino-1-naphthol, a novel 1,5-naphthalene derivative matrix suitable for matrix-assisted laser desorption/ionization in-source decay of phosphorylated peptides. Rapid Commun. Mass Spectrom. 27: 103-108, 2013. 